Proceedings of the Edinburgh Mathematical Society (2006) 49, 683-699 (C)

DOI:10.1017/S0013091505000542 Printed in the United Kingdom

\title{
THE BOUSFIELD-KAN SPECTRAL SEQUENCE FOR MORAVA $K$-THEORY
}

\author{
JOSÉ LA LUZ \\ Department of Mathematics, University of Puerto Rico, PO Box 23355, \\ San Juan 00931-3355, Puerto Rico (jluz@cnnet.upr.edu)
}

(Received 15 April 2005)

\begin{abstract}
We construct a spectral sequence converging to the $E_{2}$-term of the Bousfield-Kan spectral sequence (BKSS) for a wide variety of homology theories. Using this, the $E_{2}$-term of the BKSS based on $K(1)$-theory for the odd spheres is computed and the unstable $K(1)$-completion is computed.
\end{abstract}

Keywords: homotopy groups; Bousfield-Kan spectral sequence; unstable Adams spectral sequence

2000 Mathematics subject classification: Primary 55T15; 55Q52

\section{Introduction}

Given a homology theory $E$, we can construct the Bousfield-Kan spectral sequence (BKSS). This spectral sequence computes, for a given space $X$, the homotopy of an appropriate completion from $E_{*}(X)$. Even though we can set this spectral sequence with great generality, the $E_{2}$-term turns out to be an Ext group in some non-abelian category. In practical terms, this description limits our ability to make computations. By requiring that $E$ be a Landweber exact homology theory, and with some mild assumptions on the space $X$, we can relate the $E_{2}$-term to an Ext group in an abelian category, which in turn can be calculated as the homology of some sub-complex of the stable cobar complex. Although many theories do not satisfy this property, we were able to construct a spectral sequence converging to the $E_{2}$-term of the BKSS. The input to this spectral sequence can again be calculated as the homology of some unstable cobar complex. In the case of $K(1)$ and for any space $X$ such that $K(1)_{*}(X)$ is cofree as a coalgebra, the main result implies that the unstable coalgebra description of $E_{2}$ is isomorphic to an unstable $K(1)_{*}(K(1))$-comodule description. As observed by Kuhn [12], this turns out to be isomorphic to the stable $E_{2}$-term. With this and the proof of convergence of the stable Adams spectral sequence, we prove convergence of the unstable analogue to the unstable $K(1)$-completion of the odd spheres. Finally, using the tower constructed by Farjoun [10] and our results, we provide an example of a finite $\mathrm{H}$-space such that either the inverse limit of this tower is not the $K(1)$-localization or the map between this tower and the tower of the BKSS does not have a left inverse. 
Hypothesis 1.1. We assume that all primes are odd and all spectra are multiplicative $\Omega$-spectra.

Notation 1.2. If $A$ is a ring, then $\operatorname{char}(A)$ will denote the characteristic of $A$ and $A\left(n_{1}, n_{2}, \ldots, n_{k}\right)$ will denote a free $A$-module generated by elements in dimensions $n_{1}, n_{2}, \ldots, n_{k}$. The $p$-adic integers will be denoted by $\mathbb{Z}_{p}^{\wedge}$. If $E$ is an $\Omega$-spectrum, then $E_{n}$ denotes the $n$th space of the $\Omega$-spectrum. The category of topological spaces, the associated homotopy category and the category of $E_{*}$-modules will be denoted by $\mathcal{T}$, $\mathcal{H O}$ and $\mathcal{A}$, respectively.

\section{The construction of the Bousfield-Kan spectral sequence}

Let $X$ be a space. We define a functor from $E: \mathcal{H O} \rightarrow \mathcal{H O}$ as follows. Define a space such that

$$
E(X)=\Omega^{\infty}\left(E \wedge \Sigma^{\infty} X\right) .
$$

If $X=S^{n}$, then $E\left(S^{n}\right)=\underline{E}_{n}$, the $n$th space in the $\Omega$-spectrum of $E$. It is easy to see that if $E$ is a free $E_{*}$-module with generators $x_{i}$, then $E(X)=\prod \underline{E}_{\left|x_{i}\right|}$. For any space $X$ and for $n \geqslant 0$, we also have

$$
\pi_{n}(E(X)) \cong E_{n}(X)
$$

where the right-hand side is a reduced $E$ homology.

The composition $\eta: X \rightarrow \Omega^{\infty} \Sigma^{\infty} X \rightarrow E(X)$ induces the $E_{*}$ Hurewicz map. Taking the homotopy fibre of this map, we get the fibre sequence $D(X) \rightarrow X \stackrel{\eta}{\rightarrow} E(X) . D$ is a functor on $\mathcal{H O}$. We inductively define $D^{n+1}(X)=D\left(D^{n}(X)\right)$. There is a tower

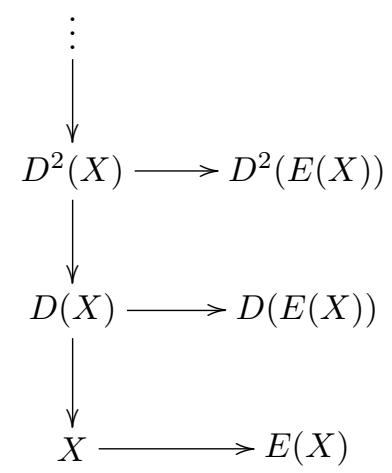

which fits into an exact couple that induces a spectral sequence with

$$
E_{1}^{s, t}=\pi_{t-s}\left(D^{s}(E(X))\right) \quad \text { for } t-s \geqslant 0
$$

and zero otherwise. We call this spectral sequence the Bousfield-Kan spectral sequence. Next, we give a description of the $E_{2}$-term. 


\section{A cosimplicial description of the $E_{2}$-term}

The following definitions will allow us to describe the $E_{2}$-term as the cohomotopy of some cosimplicial group.

Definition 3.1. A cosimplicial object $\boldsymbol{X}$ over a category $\mathcal{C}$ is a collection of objects $X_{i} \in \mathcal{C}, n \geqslant 0$, such that for each $0 \leqslant n$ there are maps $d^{i}: X_{n} \rightarrow X_{n+1}$ and $s^{i}: X_{n+1} \rightarrow$ $X_{n}$ with $0 \leqslant i \leqslant n$ satisfying the following identities:

$$
\begin{aligned}
& d^{j} d^{i}=d^{i} d^{j-1}, \quad i<j, \\
& s^{j} d^{i}= \begin{cases}d^{i} s^{j-1}, & i<j, \\
i d, & i=j, j+1, \\
d^{i-1} s^{j}, & i>j+1,\end{cases} \\
& s^{j} s^{i}=s^{i-1} s^{j}, \quad i>j .
\end{aligned}
$$

In our case the category $\mathcal{C}$ will be the category $\mathcal{A}, \mathcal{T}$ or $\mathcal{H O}$.

Given a cosimplicial object $\boldsymbol{X}$ over $\mathcal{C}$ and a functor $F: \mathcal{C} \rightarrow \mathcal{A}$, we get a cosimplicial object over the category of abelian groups. We get a cochain complex $(F(\boldsymbol{X}))$ with $\delta^{n}=\sum_{i=0}^{n}(-1)^{i} d^{i}$. In the case that $\mathcal{C}=\mathcal{H O}$ and $F=\pi_{*}$, we call the homology of this cosimplicial group the cohomotopy of $\pi_{*}(\boldsymbol{X})$ and denote it by $\pi^{*} \pi_{*} \boldsymbol{X}$.

The functor $E: \mathcal{H O} \rightarrow \mathcal{H O}$ of $\S 2$ induces a cosimplical object over $\mathcal{H O}$. We use the following definition.

Definition 3.2. A triple $(G, \mu, \eta)$ over the category $\mathcal{C}$ is composed of a functor $G$ : $\mathcal{C} \rightarrow \mathcal{C}$ and natural transformations $\mu: G^{2} \rightarrow G$ and $\eta: 1 \rightarrow G$ such that we have the following commutative diagrams:
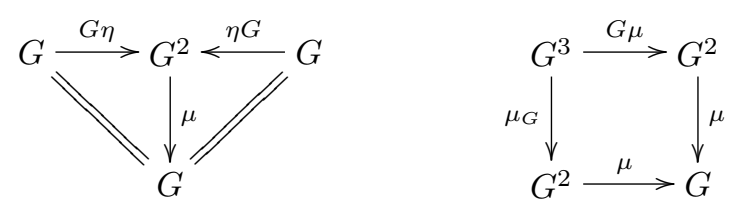

Using the triple $(G, \mu, \eta)$ we can construct a functor $\boldsymbol{G}$ from $\mathcal{C}$ to the category of cosimplicial objects over $\mathcal{C}$ as follows: let $X \in \mathcal{C}$ and define $\boldsymbol{G}(X)_{n}=G^{n+1}(X)$ and the maps

$$
d^{i}=G^{i} \eta G^{n-i}: G^{n}(X) \rightarrow G^{n+1}(X) \quad \text { and } \quad s^{i}=G^{i} \mu G^{n-i}: G^{n+2}(X) \rightarrow G^{n+1}(X)
$$

with $0 \leqslant i \leqslant n$.

The natural transformation $\mu: E^{2} \rightarrow E$, induced by the multiplicative structure of $E$, together with the Heurewitz map $\eta$, makes the functor $(E, \mu, \eta)$ a triple in the category $\mathcal{H O}$. This in turn gives us a functor $\boldsymbol{E}$ from $\mathcal{H O}$ into cosimplicial objects over $\mathcal{H O}$.

Theorem 3.3. Let $X \in \mathcal{T}$. Then

$$
E_{2}^{s, t}(X) \cong \pi^{s} \pi_{t} \boldsymbol{E}(X)
$$

Proof. The proof of this theorem can be found in $[\mathbf{9}]$. 


\section{The category $\mathcal{M}(G)$ and an alternative description of the $E_{2}$-term}

There is an alternative description of the $E_{2}$-term using the dual concept of triple. The advantage of this description is that it will enable us to describe the $E_{2}$-term as the target of a spectral sequence.

Definition 4.1. A cotriple $(G, \delta, \varepsilon)$ in a category $\mathcal{C}$ is a composed of functor $G: \mathcal{C} \rightarrow \mathcal{C}$ and natural transformations $\delta: G \rightarrow G^{2}$ and $\varepsilon: G \rightarrow 1$ such that the following diagrams commute:
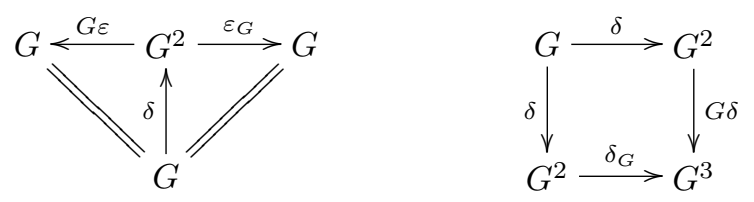

Given a cotriple $G$, a $G$-coalgebra is an object $C \in \mathcal{C}$ and a map $\psi: C \rightarrow G(C)$ such that the following diagrams commute:
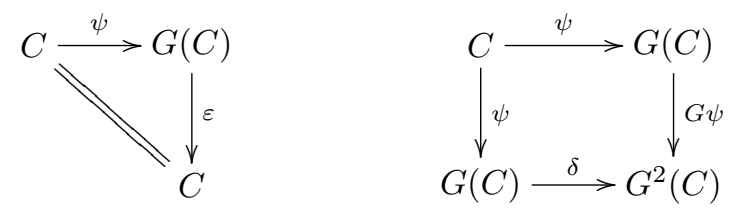

A map $f: C \rightarrow D$ is a $G$-coalgebra map if the following diagram commutes:

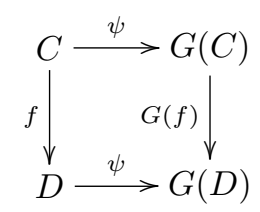

We denote the category of $G$-coalgebras over $\mathcal{C}$ as $\mathcal{C}(G)$. If $C \in \mathcal{C}$, then $G(C)$ is a $G$-coalgebra with $\psi=\delta$. Given a $G$-coalgebra $(Z, \psi)$, we can define a triple by setting

$$
\begin{aligned}
& \mu=G(\varepsilon): G^{2}(Z) \rightarrow G(Z), \\
& \eta=\psi: Z \rightarrow G(Z) .
\end{aligned}
$$

Let $\mathcal{M}$ be the category of free $E_{*}$-modules. We define a cotriple $(G, \delta, \varepsilon)$ over $\mathcal{M}$. But first we impose the following restrictions on $E$.

Hypothesis 4.2. We make the following assumptions.

(i) $E$ is a multiplicative, associative, homotopy commutative, $\mathrm{CW}$ spectrum with unit.

(ii) $E_{*}\left(E_{k}\right)$ is a free $E_{*}$-module for all $k$.

Let $M \in \mathcal{M}$ and let $F$ be the spectrum such that $\pi_{*}(F)=M$. Define $G(M)=$ $E_{*}\left(\Omega^{\infty} F\right)$. By $[\mathbf{3}]$ we know that this defines a triple over $\mathcal{M}$. With this triple we have 
the category of $G$-coalgebras over $\mathcal{M}$, or $\mathcal{M}(G)$. For $M \in \mathcal{M}(G)$ there is a resolution

$$
G(M) \stackrel{\substack{d^{0} \\ \stackrel{d^{1}}{\longrightarrow}}}{\longrightarrow} G^{2}(M) \stackrel{\stackrel{d^{0}}{\longrightarrow}}{\stackrel{d^{1}}{\longrightarrow}} \quad \ldots
$$

with codegeneracies $s^{i}$ that come from the product structure of the spectrum $E$. We call this the $G$-resolution of $M$. When we talk about the $G$-resolution of $X$ we mean the $G$-resolution of $E_{*}(X)$. Let $G(X)=G\left(E_{*}(X)\right)$.

Applying $\operatorname{Hom}_{\mathcal{M}(G)}\left(E_{*}\left(S^{t}\right),-\right)$ to the $G$-resolution of $X$ and taking the homology of this complex gives $\operatorname{Ext}_{\mathcal{M}(G)}^{s}\left(E_{*}\left(S^{t}\right), E_{*}(X)\right)$. We write $\operatorname{Ext}_{\mathcal{M}(G)}^{s, t}\left(E_{*}(X)\right)$ in place of $\operatorname{Ext}_{\mathcal{M}(G)}^{s}\left(E_{*}\left(S^{t}\right), E_{*}(X)\right)$.

Theorem 4.3. Suppose that $E$ satisfies Hypothesis 4.2 and let $X$ be a simply connected space such that $E_{*}(X) \in \mathcal{M}$. Then

$$
E_{2}^{s, t}=\operatorname{Ext}_{\mathcal{M}(G)}^{s, t}\left(E_{*}(X)\right), \quad t>s \geqslant 0 .
$$

This is proven in $[\mathbf{2}]$. The authors impose an additional condition on the spectrum $E$ (the primitives of $E_{*}\left(\underline{E}_{k}\right)$ inject into $E_{*}(E)$ ). But this condition, by [11], is not really necessary for the previous theorem.

In practice, the previous characterizations of the $E_{2}$-term are of little use. The problem is that it does not provide an explicit way to produce elements. If we assume that $E$ is a Landweber exact homology theory, like $E(n), K$ and $B P$, then we can express the $E_{2}$-term as the homology of some sub-complex of the stable cobar complex (see $[\mathbf{2}]$ ).

We would like to study the BKSS based on Morava K-theories. Unfortunately, these are not Landweber exact. In these cases a more complicated object is needed to extract information about the $E_{2}$-term.

\section{The $f$-primitive functors}

Let $\mathcal{C O}$ be the subcategory of $\mathcal{M}$ consisting of (graded) coalgebras without unit. For $n \geqslant 0$, let $R^{n} P$ be the derived functor of the primitives functor [5]. If $f: C \rightarrow D$ is a coalgebra map, we write $\left[f_{*}\right]_{i}$ for $R^{i} P(f)$.

Definition 5.1. Suppose $f: C \rightarrow C$ is a coalgebra map. Then

$$
R^{i} P_{f}(C)=\operatorname{coker}\left(\left[f_{*}\right]_{i}\right) .
$$

We call these the $f$-primitive functors.

Lemma 5.2. Let $\left\{C_{\alpha}\right\}$ be a collection of coalgebras and $f_{\alpha}: C_{\alpha} \rightarrow C_{\alpha}$ be coalgebra maps. Then

(i) the map $R^{i} P\left(C_{\alpha}\right) \rightarrow R^{i} P_{f_{\alpha}}\left(C_{\alpha}\right)$ is onto for all $i$;

(ii) $R^{i} P_{f}\left(\otimes C_{\alpha}\right)=\oplus R^{i} P_{f_{\alpha}}\left(C_{\alpha}\right)$, where $f=\otimes f_{\alpha}$. 
Proof. Part (i) follows from the definitions. Since $R^{i} P$ takes tensor products to direct sums and $\operatorname{coker}\left(\oplus f_{\alpha}\right) \cong \oplus \operatorname{coker}\left(f_{\alpha}\right)$, part (ii) follows.

Definition 5.3. We say that $C$ is $f$-nice if $R^{i} P_{f}(C)=0$ for $i>1$.

If $f$ is the zero map, then $f$-nice is just nice in the sense of [5]. It follows from the definitions that if $C$ is nice, then it is $f$-nice for all $f$.

Suppose $\operatorname{char}(A)=p$ and that $C$ is a Hopf algebra over $A$. Then the $p$ th-power map (the Frobenius) $\pi$ is a coalgebra map. Let $C\left(x_{n}\right)$ be the coalgebra with a single generator of degree $n$ and $T\left(x_{2 n}\right)$ be the coalgebra with generators $x_{2 n i}$ for $i \geqslant 1$ and

$$
\Delta\left(x_{2 n m}\right)=\sum_{i+j=m}\left(\begin{array}{c}
i+j \\
i
\end{array}\right) x_{2 n i} \otimes x_{2 n j} .
$$

This latter coalgebra is dual to a divided power algebra. We can calculate the $\pi$-primitive functors of the coalgebras $C\left(x_{n}\right)$ and $T\left(x_{2 n}\right)$ of [4]. Let $D$ be a cofree cocommutative coalgebra.

Lemma 5.4. Suppose $A$ is a ring of characteristic $p$ and $C$ is a Hopf algebra over $A$. Then:

(i) $R^{i} P_{\pi}\left(C\left(x_{2 n}\right)\right)=R^{i} P\left(C\left(x_{2 n}\right)\right)$ for all $i$;

(ii) $R^{0} P_{\pi}\left(T\left(x_{2 n}\right)\right)=A\left(x_{2 n}\right), R^{1} P_{\pi}\left(T\left(x_{2 n}\right)\right)=A\left(x_{2 n p}\right)$ and $R^{i} P_{\pi}\left(T\left(x_{2 n}\right)\right)=0$ for $i>0$;

(iii) $R^{i} P_{\pi}(D)=R^{i} P(D)$ for $i>0$.

Proof. Since $\pi(x)=0$ in $C\left(x_{n}\right)$, the induced map is zero. This gives the first case.

For the second case, let $B\left(x_{2 n}\right)$ be the coalgebra of the bipolynomial algebra with generators $\left\{x_{2 n p^{s}} \mid s \geqslant 0\right\}$ with $x_{2 n}^{p^{s}}$ primitive. The only non-trivial derived functor of this coalgebra is the zero-derived functor. This is just the module of primitives of $B\left(x_{2 n}\right)$ which gives $R^{0} P\left(B\left(x_{2 n}\right)\right)=A(2 n, 2 n p, \ldots)$. There is an injective extension sequence $T\left(x_{2 n}\right) \rightarrow B\left(y_{2 n}\right) \rightarrow B\left(z_{2 n p}\right)$. We have the following commutative diagram:

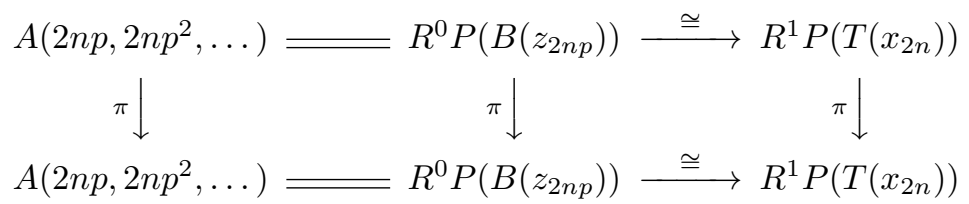

where the horizontal isomorphisms come from the proof of [4, Proposition 3.3(iv), §3]. The vertical map on the left takes the generator of dimension $2 n p^{s}$ to the generator of dimension $2 n p^{s+1}$. The result follows by taking cokernels.

For (iii), recall that if $D$ is cofree, then $R^{i} P(D)=0$ for $i>0$.

Definition 5.5. Suppose that $E_{*}\left(E_{n}\right)$ is a coalgebra for all $n$. We say $E$ is $f$-nice if $E_{*}\left(E_{n}\right)$ is $f$-nice for all $n$. 
Suppose that $\operatorname{char}\left(E_{*}\right)=p$ and there is a Kunneth isomorphism

$$
E_{*}\left(\prod \underline{E}_{n_{\alpha}}\right) \cong \otimes E_{*}\left(\underline{E}_{n_{\alpha}}\right) .
$$

Then $E_{*}\left(E_{n}\right)$ is a Hopf algebra for all $n$ and the Frobenius is a well-defined map on $E_{*}\left(E_{n}\right)$.

We would like to apply the $P_{f}$-derived functors to the $G$-resolution of $X$. But, $d^{0}$ may not commute with $f$. Fortunately, since for any $X$ we have $G(X) \cong \bigotimes_{n \in \Sigma} E_{*}\left(\underline{E}_{n}\right)$, it is sufficient to require that the following hypothesis is satisfied.

Hypothesis 5.6. $d^{0}(f(x)) \in \operatorname{Im}(f)$, where $d^{0}: G^{n}(X) \rightarrow G^{n+1}(X)$ for all $n \geqslant 0$.

From now on we assume that the homology theory $E$ satisfies Hypothesis 5.6. In this case, $f$ induces a cosimplicial map from the $G$-resolution of $X$ to itself. With this we can apply all previous results on the $P_{f}$ derived functors.

Definition 5.7. Let $R_{q}^{s} P_{f}(X)$ be the homology of the following cochain complex:

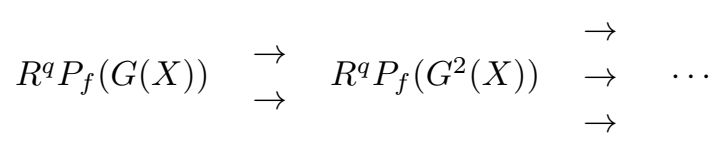

(the $s$ homology of the $q$ derived functors of the $f$-primitives applied to the $G$-resolution of $X)$.

We are interested in the case where $f$ is the Frobenius. In this case, Hypothesis 5.6 can be rephrased as follows: $d^{0}$ takes $p$ th powers to $p$ th powers. In this case there is a condition on the spaces in the $\Omega$-spectrum that will guarantee that Hypothesis 5.6 is satisfied.

Lemma 5.8. Let $\sigma: E_{*}\left(\underline{E}_{m}\right) \rightarrow E_{*}(E)$ be the stabilization map. Suppose $\operatorname{ker}(\sigma)$ is the set of decomposable elements and $x^{p}$ is primitive if and only if $x$ is primitive. Then Hypothesis 5.6 is satisfied.

Proof. Let $I_{n}=Q G^{n}(M)$ for $n \geqslant 0$. Recall that $d^{0}=\eta_{*}$. Since the stabilization map $\sigma$ commutes with differentials, we have

$$
\sigma\left(d^{0}(x y)\right)=d^{0}(\sigma(x y))=0,
$$

and by hypothesis we have $d^{0}(x y) \in I_{n+1}^{2}$. Now, if $x$ is primitive, $\pi(x)$ is primitive. Since $d^{0}$ is a coalgebra map, $d^{0}\left(x^{p}\right)$ is decomposable and primitive. But the only decomposable primitives are of the form $y^{p}$ for some primitive $y$.

\section{The (generalized) composite functor spectral sequence}

We can define a map $\sigma_{\pi}: P_{\pi} E_{*}\left(\underline{E}_{n}\right) \rightarrow E_{*}(E)$ as follows: let $x \in P_{\pi} E_{*}\left(\underline{E}_{n}\right)$ and suppose that $x^{\prime}$ is a representative in $P E_{*}\left(\underline{E}_{n}\right)$. Then $\sigma_{\pi}(x)=\sigma\left(x^{\prime}\right)$. Since $\sigma$ kills decomposable elements, $\sigma_{\pi}$ is well defined. We impose the following condition. 
Hypothesis 6.1. The map $\sigma_{\pi}$ is injective.

Remark 6.2. This is true if the hypotheses of Lemma 5.8 are satisfied.

Theorem 6.3. Suppose that Hypothesis 6.1 is satisfied and let $U_{\pi}=P_{\pi} G$. Then $U_{\pi}$ is the functor of a cotriple on $\mathcal{M}$.

Proof. In $[\mathbf{3}, \S 7]$, the authors define a cotriple $(T, \delta, \varepsilon)$ on $\mathcal{M}$ using the structure of the Hopf algebroid $\left(E_{*}, E_{*}(E)\right)$. The stabilization map induces a map of cotriples $G \stackrel{\sigma}{\rightarrow} T$. From $\S 5$ we know that if $(M, \psi)$ is a $G$-coalgebra, then $M$ is an $E_{*}(E)$-comodule and $P(M)$ is a sub- $E_{*}(E)$-comodule of $M$ with map $\Psi: P(M) \rightarrow E_{*}(E)$. We have an induced $E_{*}(E)$-comodule $P_{\pi}(M)$. Let $\Psi_{\pi}$ be the induced comodule map, $j: P(M) \rightarrow P_{\pi}(M)$ be the projection and $i: P(M) \rightarrow M$ be the injection. Then we have a commutative diagram:

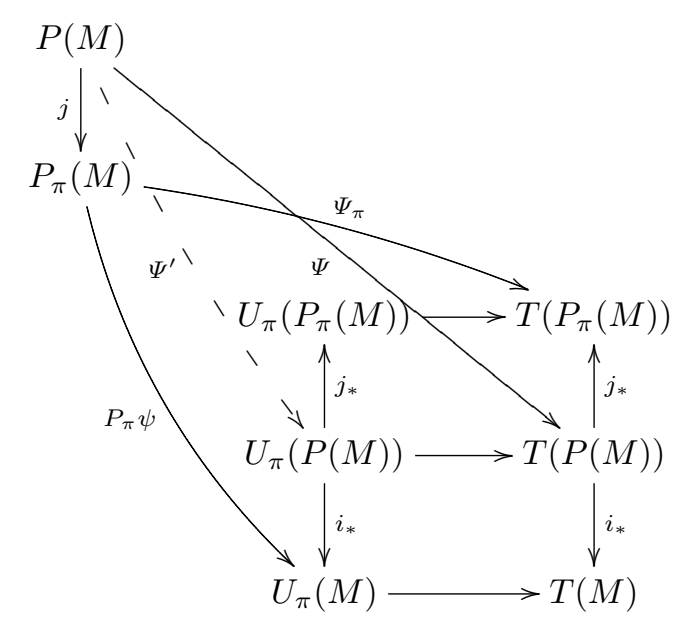

where $i_{*}$ is an injection, the rightmost $j_{*}$ is a surjection and the horizontal maps are injections by Hypothesis 6.1. Since $\Psi$ is induced from $\sigma$, there is a well-defined map $\psi_{\pi}: P_{\pi}(M) \rightarrow U_{\pi}\left(P_{\pi}(M)\right)$. If we now let $M=G(N)$ for $N \in \mathcal{M}$, we have a map $\delta_{\pi}: U_{\pi}(N) \rightarrow U_{\pi}^{2}(N)$. We let the composition $U_{\pi}(N) \rightarrow T(N) \stackrel{\varepsilon}{\rightarrow} N$ be $\varepsilon_{\pi}$. Since $U_{\pi} \rightarrow T$ is an injection that commutes with the cotriple structure of $T,\left(U_{\pi}, \delta_{\pi}, \varepsilon_{\pi}\right)$ is a cotriple.

With this result we can construct the category of $U_{\pi}$-coalgebras and state the following theorem.

Theorem 6.4. Suppose that the homology theory E satisfies Hypotheses 5.6 and 6.1. There is then a spectral sequence

$$
\bar{E}_{2}^{m, n, t}=\operatorname{Ext}_{U_{\pi}}^{m, t}\left(R_{0}^{n} P_{\pi}(X)\right) \Rightarrow E_{2}^{n+m, t}(X)
$$

(converging to the $E_{2}$-term of the BKSS based on E-theory). 
Proof. Fix a $t \geqslant 0$. We form, for $n, m \geqslant 0$, the double complex concentrated at degree $t$ :

$$
D^{m, n, t}=D^{m, n}(X)_{t}=U_{\pi}^{n} P_{\pi} G^{m+1}(X)_{t} .
$$

For each fixed $n$ we have

$$
D^{*, n, t}=D^{*, n}(X)_{t}=U_{\pi}^{n} P_{\pi}\left(\boldsymbol{G}(X)_{t}\right),
$$

where the maps are induced by the $G$-resolution of $X$. Next we fix $m$. We have

$$
D^{m, *, t}=D^{m, *}(X)_{t}=\boldsymbol{U}_{\boldsymbol{\pi}}\left(P_{\pi} G^{m+1}(X)\right)_{t} .
$$

This is just the $P_{\pi}$-complex for $P_{\pi} G^{m}(X)$. Fixing $m$ and taking homology we get

$$
\bar{E}_{1}^{m, n, t}=\operatorname{Ext}_{U_{\pi}}^{m, t}\left(U_{\pi}\left(G^{m}(X)\right)\right)= \begin{cases}G^{m}(X)_{t} & \text { if } n=0, \\ 0 & \text { otherwise. }\end{cases}
$$

Taking homology again we have $E_{2}^{m, t}(X)$. If we fix $n$ and take homology, we find that

$$
\bar{E}_{1}^{m, n, t}=U_{\pi}^{m} R_{0}^{n} P_{\pi}(X)_{t},
$$

and taking homology again we find the following:

$$
\bar{E}_{2}^{m, n, t}=\operatorname{Ext}_{U_{\pi}}^{m, t}\left(R_{0}^{n} P_{\pi}(X)\right) .
$$

This tells us that knowledge of the functors $R_{0}^{m} P_{\pi}(X)$ is sufficient to give us the $E_{2}$ term of the BKSS based on $E$. We now need information about these objects. We have the following theorem, which gives us a way to approach these objects.

Theorem 6.5. Suppose that the homology theory E satisfies Hypotheses 5.6 and 6.1. There is then a spectral sequence

$$
E_{2}^{i, j}=R_{i}^{j} P_{\pi}(X) \Rightarrow R^{i+j} P_{\pi}\left(E_{*}(X)\right) .
$$

Proof. Consider the following double complex:

$$
D^{i, j}=P_{\pi} S^{j+1} G^{i+1}(X) .
$$

Fixing $i$ first, we have

$$
D^{i, *}=P_{\pi} \tilde{\boldsymbol{S}}\left(G^{i+1}(X)\right) .
$$

This is just the functor $P_{\pi}$ applied to the $S$-resolution for $G^{i+1}(X)$.

Now fixing $j$, we have

$$
D^{*, j}=P_{\pi} S^{j+1} \boldsymbol{G}(X) .
$$

This map is induced by the $G$-resolution of $M$. Fixing $i$ again and taking homology, we have

$$
E_{1}^{i, j}= \begin{cases}R^{i+1} P_{\pi}\left(G^{j+1}(X)\right) & \text { if } i=j, \\ 0 & \text { otherwise. }\end{cases}
$$


Taking homology again we have $R_{i}^{j} P_{\pi}(X)$. Let us now fix $j$. We get

$$
E_{1}^{i, j}= \begin{cases}P_{\pi} S^{j+1}\left(E_{*}(X)\right) & \text { if } i=0, \\ 0 & \text { otherwise }\end{cases}
$$

Homology again gives $R^{j} P_{\pi}\left(E_{*}(X)\right)$.

Corollary 6.6. Suppose that the homology theory E satisfies Hypotheses 5.6 and 6.1. Suppose also that $E$ is $\pi$-nice. There is then a long exact sequence

$$
\cdots \rightarrow R_{0}^{k} P_{\pi}(X) \rightarrow R^{k} P_{\pi}\left(E_{*}(X)\right) \rightarrow R_{1}^{k-1} P_{\pi}(X) \rightarrow R_{0}^{k+1} P_{\pi}(X) \rightarrow \cdots .
$$

Proof. Since $E_{*}\left(E_{k}\right)$ is nice, and since $G^{r}(X)$ is just a tensor product of these Hopf algebras, for $k>1$, we have $R_{k}^{j} P_{\pi}(X)=0$. We get a spectral sequence with just two rows. This becomes a long exact sequence.

Theorem 6.7. Suppose that $R^{q} P_{\pi}\left(E_{*}(X)\right)=0$ for $q \geqslant n$ :

(i) $R_{0}^{0} P_{\pi}(X) \cong P_{\pi}\left(E_{*}(X)\right)$,

(ii) $R_{0}^{q} P_{\pi}(X) \cong R_{1}^{q+2} P_{\pi}(X)$ for $q \geqslant n$,

(iii) $R_{0}^{1} P_{\pi}(X)$ injects into $R^{1} P_{\pi}\left(E_{*}(X)\right)$ and, if $E_{*}(X)$ is cofree, then $R_{0}^{1} P_{\pi}(X)$ is trivial.

Proof. This follows from Corollary 6.6.

Remark 6.8. If $f$ is the zero map, then $P_{f}=P$, and Hypothesis 6.1 is merely the requirement that $\sigma$ is injective on the primitives. This is satisfied by all $p$-local Landweber theories. Also, Hypothesis 5.6 is not necessary if $E$ is Landweber.

\section{The Morava $K$-theory case}

We want to apply the previous results to $K(n)$. But first we need to make sure that Hypotheses 5.6 and 6.1 are satisfied. By Lemma 5.8 and Remark 6.2 it is sufficient that $\operatorname{ker}(\sigma)$ is the set of decomposable elements and that the only primitives are $x$ and the $p$ th powers of $x$ where $x$ is primitive.

Lemma 7.1. If $E=K(n)$, then $\operatorname{ker}(\sigma)$ is the set of decomposable elements and $x^{p}$ is primitive if and only if $x$ is primitive. Also $K(n)$ is $\pi$-nice.

Proof. By [14] $\Gamma_{n, m}=K(n)_{*}\left(\frac{K(n)_{m}}{1 . T_{m}}\right)$ has generators $a^{I} \circ b_{(0)}^{j_{0}} \circ b^{J} \circ e_{1}^{\varepsilon}$ with $\varepsilon=0,1$, $i_{k}=0,1,0 \leqslant j_{k}<p^{n}, j_{0}<p^{n}-\overline{1 .}$. These elements stabilize to $\tau^{I} b^{J}$. So $\sigma$ is injective on the indecomposables. Using the Hopf algebra structure computed in [14] one can conclude that $x^{p}$ is primitive if and only if $x$ is primitive.

To prove that $K(n)$ is $\pi$-nice is sufficient to prove that each $K(n)_{*}\left(K(n)_{k}\right)$ is nice. By [14] we know that $K(n)_{*}\left(K(n)_{k}\right)$ is a tensor product of exterior, truncated and polynomial Hopf algebras. The exterior algebra only contributes zero-derived functors, the (primitively generated) polynomial algebra is a tensor product of coalgebras of the 
type $T\left(x_{2 n}\right)$ (see [4]) and this has primitive dimension 1 . So the only unknown is the truncated algebra. But by [14, Theorem 2.1] this is just a divided power algebra $\bmod p$ and this is cofree.

Remark 7.2. Hypothesis 6.1 is not satisfied by $E(1) \bmod p$. The element

$$
v_{1} b_{(0)}^{p-1} \circ b_{(1)}^{n} \circ e_{1}-b_{(1)}^{n} \circ e_{1} \circ\left[v_{1}\right] \in E(1)_{*}\left(E(1)_{2 n+1}\right)
$$

is non-zero unstably but it is in $\operatorname{ker}(\sigma)$. This does not happen in $K(1)$ because we have the extra relation $v_{1} b_{(0)}^{p-1} \circ b_{(1)}^{n} \circ e_{1}=b_{(1)}^{n} \circ e_{1} \circ\left[v_{1}\right]$.

\section{Applications to $K(1)$}

We apply all the previous results to $K(1)$. In this case we can say much more.

Corollary 8.1. Suppose that $R^{i} P_{\pi}\left(K(1)_{*}(X)\right)=0$ for $i \geqslant n$. Then, for $i \geqslant n+1$,

$$
R_{0}^{i} P_{\pi}(X)=0 .
$$

Proof. Suppose $i>n+1$ and $R_{0}^{i} P_{\pi}(X) \neq 0$ and we have a generator $x$. Since we can get new generators from $x$ by multiplying by $v_{1}^{k}$, we consider $R^{1} P_{\pi}\left(G^{i+2}(X)\right) \otimes \mathbb{Z}_{p}$. By the universal coefficients theorem and Lemma 7.1, we know that the only part contributing first-derived functors is the polynomial part. This is of the form $\otimes_{\alpha \in \Lambda} T\left(x_{2 n_{\alpha}}\right)$ for some index set $\Lambda$. The generator $x_{2 n_{\alpha}}$ is of the form $a_{0} \circ b^{J_{\alpha}}$ with dimension $2\left(1+\sum j_{i_{\alpha}} p^{i}\right)$ with $j_{0}<p-1$ and $n_{\alpha}=1+\sum j_{i} p^{i}$. Since $R^{1} P\left(T\left(x_{2 n_{\alpha}}\right)\right)=\mathbb{Z}_{p}\left(2 n_{\alpha} p\right)$, we have generators of degree $2 p\left(1+\sum j_{i_{\alpha}} p^{i}\right)$. Now let us look at generators for

$$
R^{0} P_{\pi}\left(G^{i+1}(X)\right) \otimes \mathbb{Z}_{p}=P_{\pi}\left(G^{i+1}(X)\right) \otimes \mathbb{Z}_{p}
$$

This has generators $a_{(0)} \circ b^{K} \circ e_{1}^{\varepsilon}$ and $b_{(0)} \circ b^{K} \circ e_{1}^{\varepsilon}$ of degree $2\left(1+\sum_{k \geqslant 0} k_{i} p^{i}\right)+\varepsilon$ with $k_{0}<p-1$ in both cases. By Theorem 6.7(i) the dimensions of the generators have to agree. So $\varepsilon=0$. But the generators of the first case were divisible by $p$ and neither of the other two are.

In fact this result implies the following theorem.

Theorem 8.2. Let $X$ be an $H$-space and suppose that $K(1)_{*}(X)$ is cofree. Then

$$
E_{2}^{s, t}(X) \cong \operatorname{Ext}_{U_{\pi}}^{s, t}\left(P_{\pi}\left(K(1)_{*}(X)\right)\right) .
$$

Proof. Since $K(1)_{*}(X)$ is cofree, by 8.1 we know that we have only zero-derived functors of $P_{\pi}$, and so the spectral sequence of Theorem 6.4 collapses and the result follows.

Corollary 8.3. Suppose that $K(1)_{*}(X)$ is cofree. Then

$$
E_{2}(X) \cong E_{2}^{\mathcal{S}}\left(\Sigma^{\infty} X\right)
$$

where the object on the right is the $E_{2}$-term of the stable Adams spectral sequence. 
Proof. The category $U_{\pi}$ is just the category of unstable $K(1)_{*}(K(1))$-comodules. By [12], there are no unstable $K(n)_{*}(K(n))$-comodules. Since we have a collapse to the zero line of the spectral sequence of Theorem 6.4, then the two $E_{2}$-terms have to agree.

Remark 8.4. From the previous corollary, the $E_{2}$-terms of the stable spectral sequence for the sphere and the unstable spectral sequence for the odd sphere agree. This does not happen with the even sphere. By Lemma $5.4, R^{1} P_{\pi}\left(S^{2 n}\right)$ is a $K(1)_{*}$-module with a generator in dimension $4 n$. This leads to a long exact sequence:

$$
\cdots \rightarrow \operatorname{Ext}_{U_{\pi}}^{s, t}\left(S^{2 n}\right) \rightarrow \operatorname{Ext}_{\mathcal{M}(G)}^{s, t}\left(S^{2 n}\right) \rightarrow \operatorname{Ext}_{U_{\pi}}^{s-1, t}\left(S^{4 n}\right) \rightarrow \cdots
$$

where the last map on the left has bidegree $(2,0)$.

We now develop a way of computing $\operatorname{Ext}_{U_{\pi}}(M)$ for all $K(n)$. With this we can calculate specific elements in the $K(n)$-based spectral sequence.

\section{Calculation of $U_{\pi}(M)$ for Morava $K$-theories}

Our knowledge of $\operatorname{Ext}_{U_{\pi}}(M)$ depends on $U_{\pi}^{n}(M)$ and the differentials.

From $[\mathbf{1 5}]$ we know that

$$
K(n)_{*}(K(n))=\Lambda\left[\tau_{0}, \ldots, \tau_{n-1}\right] \otimes K(n)_{*} \otimes_{B P_{*}} \frac{B P_{*}(B P)}{\left(v_{n} t_{i}^{p^{n}}-v_{n}^{p^{i}} t_{i}\right)} .
$$

As in [3], we use the basis consisting of $h_{n}$ instead of $t_{n}$. Since the $\tau_{k}$ comes from the dual of the Steenrod algebra, this implies that the canonical anti-isomorphism is given by

$$
\tau_{k}+\sum_{i=0}^{k} t_{k-i}^{p^{i}} c\left(\tau_{i}\right)=0
$$

or $\tau_{k}=c\left(\tau_{k}\right)$ mod decomposables. Let $\beta_{k}=c\left(\tau_{k}\right)$. Then the exterior part of $\Gamma_{n}$ is generated by $\beta_{i}$ with $0 \leqslant i \leqslant n-1$.

Notation 9.1. We define

$$
<_{i}= \begin{cases}< & \text { for } i=0 \\ \leqslant & \text { for } i=1\end{cases}
$$

Theorem 9.2. Let $\Gamma_{n}=K(n)_{*}(K(n))$ and $M$ be a $K(n)_{*}$-module. Then the following conditions apply.

(i) $U_{\pi}(M)=\operatorname{span}_{K(n)_{*}}\left\{\beta^{I} h^{J} \otimes m \in \Gamma_{n} \otimes M\left|l(I)+2 l(J)<_{i_{0}}\right| m \mid\right\}$.

(ii) $U_{\pi}(M)$ injects into the stable cobar complex. 
(iii) Suppose that $M$ is an unstable $\Gamma$-comodule, with coaction $\Psi: M \rightarrow U_{\pi}(M)$. The differential is then given by

$$
\begin{array}{r}
d\left(\left[\gamma_{1}|\cdots| \gamma_{n}\right] m\right)=\left[1\left|\gamma_{1}\right| \cdots \mid \gamma_{n}\right] m+\sum_{j=1}^{n}(-1)^{j}\left[\gamma_{1}|\cdots| \gamma_{j}^{\prime}\left|\gamma_{j}^{\prime \prime}\right| \cdots \mid \gamma_{n}\right] \\
+(-1)^{n+1} \sum\left[\gamma_{1}|\cdots| \gamma_{n}^{\prime}\right] m^{\prime},
\end{array}
$$

where $\Psi\left(\gamma_{j}\right)=\sum \gamma^{\prime} \otimes \gamma^{\prime \prime}$ and $\Psi(m)=\sum \gamma^{\prime} \otimes m^{\prime}$.

Proof. The primitive elements in $K(n)_{*}\left(\underline{K(n)}_{m}\right)$ are

(i) exterior: $\left[v_{n}^{k}\right] \circ a^{I} \circ b_{0}^{j_{0}} \circ b^{J} \circ e_{1}$ with $i_{0}=1$ or $j_{0}=1$;

(ii) truncated: $\left[v_{n}^{k}\right] \circ a^{I} \circ b_{0}^{j_{0}} \circ b^{J}$ with $I \neq I(1)$ and $i_{0}=1$ or $j_{0}=1$;

(iii) polynomial: $\left(\left[v_{n}^{k}\right] \circ a^{I(1)} \circ b_{0}^{j_{0}} \circ b^{J}\right)^{* p^{k}}, k \geqslant 1$.

The cases (i) and (ii) are also indecomposable. In case (iii) the only element that is indecomposable is when $k=1$. All of the cases suspend to $\beta^{I} h^{J} \otimes v_{n}^{k} i_{m}$. We have, for all cases,

$$
l(I)+2 j_{0}+2 l(J)-k q_{n}=m
$$

or

$$
l(I)+2 l(J) \leqslant l(I)+2 j_{0}+2 l(I)=m+k q_{n}=\left|v_{n}^{k} i_{m}\right|,
$$

where $q_{n}=\left|v_{n}\right|$. The inequality on the left-hand side is strict if $j_{0}=1$ and is an equality if $i_{0}=j_{0}=1$. This proves (i).

Part (ii) follows immediately from (i). Part (iii) follows from the fact that we know the differential in the stable cobar. Since $U_{\pi}(M)$ injects, the result follows.

At last we see why we chose the derived functors of the $\pi$-primitives instead of just the usual derived functors of the primitives: these do not inject into the stable object (the $p$ th powers of $\left[v^{k}\right] \circ a_{(0)} \circ b_{(0)}^{j_{0}} \circ b^{I}$ are killed by $\left.\sigma\right)$.

Let us find some elements in $E_{2}\left(S^{2 n+1} ; K(1)\right)$. In this case we have $\beta_{0}=c\left(\tau_{0}\right)=\tau_{0}$. The only elements in $\Gamma=K(1)_{*}(K(1))$ that are primitive are $h_{1}^{p k}, k>0$, and $\tau_{0}$. But, by [15], in $\Gamma$ we have $h_{1} v_{1}^{p}=h_{1}^{p} v_{1}$, so we have only to consider $h_{1}$ and $\tau_{0}$. We therefore see that, for $M=K(1)_{*}\left(S^{2 n+1}\right), n>0, E_{2}$ is generated by $h_{1}$ and $\tau_{0}$.

From now on the element $\left[\gamma_{1}|\cdots| \gamma_{n}\right] i_{2 n+1}$ will be represented in homology by $\gamma_{1} \cdots \gamma_{n} i_{2 n+1}$ or, if it is clear on which sphere we are working, $i_{2 n+1}=1$ and $\gamma_{1} \cdots \gamma_{n}$, with the convention that $\operatorname{deg}\left(\gamma_{1} \cdots \gamma_{n}\right)=2 n+1+\sum \operatorname{deg}\left(\gamma_{i}\right)$. Since for $K(1)$ the right action and the left actions commute, we immediately have

$$
E_{1}^{0, t}\left(S^{2 n+1}\right)= \begin{cases}\mathbb{Z}_{p} & \text { if } t=2 n+1+k q, k \in \mathbb{Z} \text { generated by } v_{1}^{k}, \\ 0 & \text { otherwise. }\end{cases}
$$

The filtration- $r$ elements $\tau_{0}^{r}$ and $v_{1}^{k} h_{1} \tau_{0}^{r-1}$ are non-trivial. 


\section{Composition pairings in the $K(1)$ spectral sequence}

By [7], there is also composition pairing in the spectral sequence for $t-s \geqslant 1$ and $r \geqslant 2$ :

$$
E_{r}^{s, m+t}(X) \otimes E_{r}^{s^{\prime}}, t^{\prime}\left(S^{m}\right) \stackrel{\circ}{\rightarrow} E_{r}^{s+s^{\prime}, t+t^{\prime}}(X) .
$$

Given the natural map $i: X \rightarrow X_{E}^{\wedge}$, where $X_{E}^{\wedge}$ is the completion, there is a commutative diagram:

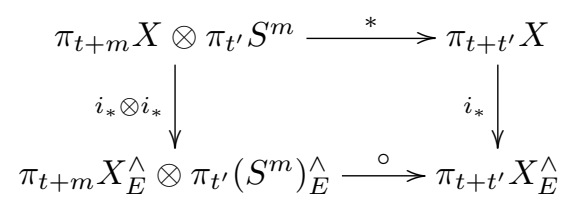

Lemma 10.1. The composition corresponds (up to sign) to the Yoneda product in the category $U_{\pi}$ :

$$
\begin{array}{r}
\operatorname{Ext}_{U_{\pi}}^{s}\left(K(1)_{*}\left(S^{t+2 m+1}\right), K(1)_{*}\left(S^{2 n+1}\right)\right) \otimes \operatorname{Ext}_{U_{\pi}}^{s^{\prime}}\left(K(1)_{*}\left(S^{t^{\prime}}\right), K(1)_{*}\left(S^{2 m+1}\right)\right) \\
\rightarrow \operatorname{Ext}_{U_{\pi}}^{s}\left(K(1)_{*}\left(S^{t+2 m+1}\right), K(1)_{*}\left(S^{2 n+1}\right)\right) \otimes \operatorname{Ext}_{U_{\pi}}^{s^{\prime}}\left(K(1)_{*}\left(S^{t+t^{\prime}}\right), K(1)_{*}\left(S^{t+2 m+1}\right)\right) \\
\rightarrow \operatorname{Ext}_{U_{\pi}}^{s+s^{\prime}}\left(K(1)_{*}\left(S^{t+t^{\prime}}\right), K(1)_{*}\left(S^{2 n+1}\right)\right) .
\end{array}
$$

Proof. This follows from [7].

We use this result to study compositions by $\tau_{0}$. The proofs of the next two results are analogous to the proof of the stable statements.

Claim 10.2. For $k>0, \tau_{0}^{k} \neq 0$.

As in the stable case, we have an element representing multiplication by $p$ in homotopy.

Lemma 10.3. Suppose that the spectral sequences for $X$ and $S^{2 n+1}$ converge. Suppose also that $x \in E_{2}$ survives to $E_{\infty}$ and represents $\alpha \in \pi_{*}(X)$. Then $a_{(0)} \circ x$ represents $p \alpha \in \pi_{*}(X)$.

Form this it follows that we have infinite towers, for $k \in \mathbb{Z}$, in dimensions $t-s=$ $2 n+1+k q$, generated by $v_{1}^{k} \tau_{0}^{s}$, and towers in dimension $t-s=2 n+k q$ generated by $v_{1}^{k} \tau_{0}^{s} h_{1}$. The only thing missing is knowledge about the differentials.

\section{Convergence of the stable Adams spectral sequence}

The next result will give us the missing piece. We define $\nu(k)$ as $k=a p^{\nu(k)}$ with $p \nmid a$.

Theorem 11.1. The stable Adams spectral sequence based on $K(1)$ of the sphere converges for $t-s>0$ and $v_{1}^{k}$ supports a $d_{\nu(k)+2}$ differential.

Proof. Since the only place in which the $E_{2}$-term has classes is in dimensions $t-s=$ $k q-1, k q$ with $k \in \mathbb{Z}$, it is sufficient to worry about those dimensions. By [13] we know that the $E(1)$ spectral sequence for the sphere converges and $\pi_{*}\left(S_{E(1)}^{\wedge}\right)$ has a $\mathbb{Z}_{p^{\nu(k)+1}}$ generated by $\alpha_{k}=d_{1}\left(v_{1}^{k}\right) / p^{\nu(k)+1}$ in dimension $k q-1$. We have a map of ring spectra 
$j: E(1) \rightarrow K(1)$, which induces a map between the spectral sequence of these spectra and sends $\alpha_{k}$ to $v_{1}^{k-1} h_{1}$. Since $E_{r}^{s, t}=0$ for any $t-s=k q-2$, the element

$$
v_{1}^{k-1} h_{1} \in \underset{s}{\lim _{s}} \pi_{k q-1}\left(\overline{K(1)}^{s}\right)
$$

and since there is an onto map to $\pi_{k q-1}\left(S_{K(1)}^{\wedge}\right)$ we have

$$
v_{1}^{k-1} h_{1} \in \pi_{k q-1}\left(S_{K(1)}^{\wedge}\right)
$$

This implies that $1 \leqslant \operatorname{ord}\left(v_{1}^{k-1} h_{1}\right) \leqslant p^{\nu(k)+1}$. Since multiplication by $\tau_{0}$ represents multiplication by $p$, the tower over $v_{1}^{k-1} h_{1}$ has to be killed at filtration less than or equal to $\nu(k)+2$. So $v_{1}^{k}$ supports a $d_{r}$ differential, where $r \leqslant \nu(k)+2$.

We prove $\nu(k)+2=r$ by induction on $d_{n}$. For $k=1$, we have, by the previous paragraph, $d_{2}\left(v_{1}\right)=\tau_{0} h_{1}$ and, using the derivation rule, we have $d_{2}\left(v_{1}^{k} \tau_{0}^{w}\right)=k v_{1}^{k-1} \tau_{0}^{w+1} h_{1}$. Suppose now that

$$
d_{m}\left(v_{1}^{k p^{m-2}} \tau_{0}^{w}\right)=k v_{1}^{k p^{m-2}-1} \tau_{0}^{w+m-1} h_{1} \quad \text { for } m<n .
$$

By the induction hypothesis we know that $d_{n-1}\left(v_{1}^{p^{n-2}}\right)=p v_{1}^{p^{n-2}-1} \tau_{0}^{n-2} h_{1}=0$. So the smallest differential in which $v_{1}^{p^{n-2}}$ is non-zero is $d_{n}=d_{\nu(n-2)+2}$. The general result follows from using the derivation rule.

Corollary 11.2. The BKSS based on $K(1)$ for the sphere converges completely and, for $m \geqslant 1$, the completion in the sense of $[\mathbf{1}]$ is given by

$$
\pi_{m}\left(\left(S^{2 n+1}\right)^{\wedge}\right)= \begin{cases}\mathbb{Z}_{p^{\nu(k)+1}} & \text { for } m=2 n+k q, k \in \mathbb{Z}-\{0\} \\ \mathbb{Z}_{p}^{\wedge} & \text { for } m=2 n+1,2 n \\ 0 & \text { otherwise. }\end{cases}
$$

Proof. The differentials on $v_{1}^{k}$ for $k \geqslant 0$ can be deduced by the stable differentials. For $k \leqslant 0,[7]$ says that $d_{r}$ is a derivation for $r>1$. We have the following formulae:

$$
0=d_{r}(1)=d_{r}\left(v_{1}^{k} v_{1}^{-k}\right)=d_{r}\left(v_{1}^{k}\right) v_{1}^{-k}+v_{1}^{k} d_{r}\left(v_{1}^{-k}\right)
$$

or $d_{r}\left(v_{1}^{-k}\right)=-v_{1}^{-2 k} d_{r}\left(v_{1}^{k}\right)$. Since we have a vanishing line, by $[\mathbf{8}, \S \mathrm{IX}]$ we know that the spectral sequence converges completely.

\section{Some remarks on the general $K(n)$ spectral sequence}

Although we have not been able to prove that the $R_{0}^{i} P_{\pi}(X)$ vanish when the groups $R^{i} P_{\pi}\left(K(n)_{*}(X)\right)$ vanish for $n>1$, we have the following theorem.

Theorem 12.1. The zero line of the spectral sequence of Theorem 6.4 for $K(n)$ injects into the stable Adams spectral sequence. 
Proof. The spectral sequence of Theorem 6.4 has an edge homomorphism:

$$
\operatorname{Ext}_{U_{\pi}}^{m, t}\left(P_{\pi} K(n)_{*}(X)\right)=\bar{E}_{2}^{m, 0, t} \rightarrow E_{2}^{m, t}(X) .
$$

Since this construction commutes with stabilization, we have a commutative diagram:

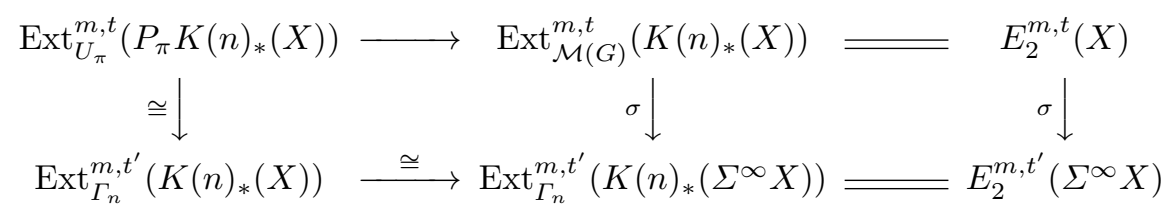

where $\Gamma_{n}=K(n)_{*}(K(n))$.

\section{The $K(1)$-completion of $S^{2 n+1}$ and its relation to the work of Farjoun}

In [10], Farjoun defines a tower $\left\{Y_{n}(X)\right\}$ under $X$ as follows. Let $E(X)$ be the functor defined in $\S 2$ and let $Y_{1}(X)=E(X)$. Define

$$
Y_{n}(X)=\mathrm{fb}\left[Y_{n-1}(X) \rightarrow E\left(\frac{Y_{n-1}(X)}{X}\right)\right],
$$

where fb denotes the homotopy fibre. The null homotopy from $X$ into the cofibre gives

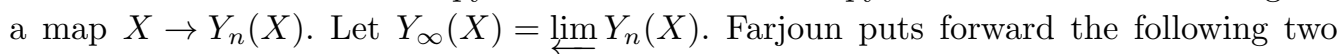
questions.

Question 13.1. Let $X$ be an H-space of finite type. Is the natural map $X_{E} \rightarrow Y_{\infty}$ a homotopy equivalence?

Question 13.2. When does the natural map of towers $\left\{Y_{n}(X)\right\}_{n \in \mathbb{N}} \rightarrow\left\{D^{n}(X)\right\}_{n \in \mathbb{N}}$ have a left inverse?

Although we cannot answer Question 13.1 in the affirmative or get necessary conditions to answer Question 13.2, we can deduce the following from our work.

Lemma 13.3. Let $E=K(1)$. Then Questions 13.1 and 13.2 cannot be true at the same time for $X=S^{2 n+1}$.

Proof. Suppose that if $X$ is an H-space of finite type, this implies that the natural map $X_{E} \rightarrow Y_{\infty}$ is a homotopy equivalence and the map of towers from Question 13.2 has a left inverse. We then have an injective map

$$
\pi_{*}\left(S_{K(1)}^{2 n+1}\right) \rightarrow \pi_{*}\left(K(1)^{\wedge}\left(S^{2 n+1}\right)\right) .
$$

The $K(1)$-localization of the odd spheres was calculated in [6]. We have

$$
\pi_{i}\left(S_{K(1)}^{2 n+1}\right)= \begin{cases}\mathbb{Z}_{p^{\nu(k)}+1} & \text { for } i=2 n+q k, 2 n-1+k q, k \in \mathbb{Z}-\{0\} \\ \mathbb{Z}_{p}^{\wedge} & \text { for } i=2 n, 2 n-1, \\ \mathbb{Z}_{p}^{\wedge} \oplus \mathbb{Z}_{p}^{\wedge} & \text { for } i=2 n-1, \\ 0 & \text { otherwise. }\end{cases}
$$


Comparing this with the result of Corollary 11.2, we see that the map cannot be injective.

Acknowledgements. The author thanks Professor Martin Bendersky. His ideas and suggestions have been of vital importance for this research.

\section{References}

1. M. Bendersky and J. R. Hunton, On the coalgebraic ring and the Bousfield-Kan spectral sequence for a Landweber exact spectrum, Proc. Edinb. Math. Soc. 47 (2004), 513-532.

2. M. Bendersky and R. D. Thompson, The Bousfied-Kan spectral sequenece for periodic homology theories, Am. J. Math. 122 (2000), 599-635.

3. M. Bendersky, E. B. Curtis And H. R. Miller, The unstable Adams spectral sequence for generalized homology, Topology 17 (1978), 229-248.

4. M. Bendersky, E. B. Curtis and D. Ravenel, EHP sequences in BP theory, Topology 21 (1982), 373-391.

5. A. K. Bousfield, Nice homology coalgebras, Trans. Am. Math. Soc. 148 (1970), 473489.

6. A. K. Bousfield, The $K$-theory localizations and $v_{1}$-periodic homotopy groups of Hspaces, Topology 38 (1999), 1239-1264.

7. A. K. Bousfield, Cosimplicial resolutions and homotopy spectral sequences in model categories, Geom. Topol. 7 (2003), 1001-1053.

8. A. K. Bousfield AND D. M. KAN, Homotopy limits, completions and localizations, Lecture Notes in Mathematics, Volume 304 (Springer, 1970).

9. A. K. Bousfield AND D. M. KAN, The homotopy spectral sequence of a space with coeficients in a ring, Topology 11 (1972), 79-106.

10. E. D. Farjoun, Two completion towers for generalized homology, Contemp. Math. 265 (2000), 27-39.

11. R. KARGL, An unstable Adams spectral sequence based on a generalized homology theory, PhD thesis, Graduate Center, City University of New York (1998).

12. N. J. Kunn, Morava K-theories and infinite loop spaces, algebraic topology, Lecture Notes in Mathematics, Volume 1370 (Springer, 1989).

13. H. R. Miller, D. C. Ravenel and W. S. Wilson, Periodic phenomena in the AdamsNovokov spectral sequence, Ann. Math. 106 (1977), 469-516.

14. W. S. Wilson, The Hopf ring for Morava K-theory, Publ. RIMS Kyoto 20 (1984), 10251036.

15. N. Yagita, A topological note on the Adams spectral sequence based on Morava's $K$ theory, Proc. Am. Math. Soc. 72 (1978), 613-617. 